\title{
LA CONEXIÓN LECTURA-ESCRITURA EN LA ENSEÑANZA DE LAS LENGUAS EXTRANJERAS
}

\author{
Carlos Navarro Thames \\ Profesor de la Escuela de Lenguas Modernas
}

Recibido 30-VIII-2004 • Aceptado 7-IX-2004

\begin{abstract}
Resumen: La interacción entre la lectura y la escritura no ha recibido toda la atención que merece en la enseñanza de las lenguas extranjeras. De igual manera, ambas destrezas han tradicionalmente enseñadas en forma separada lo cual va en detrimento de la adquisición de una destreza o ambos por parte del estudiante. Este estudio propone que entre más lea un estudiante de un segundo idioma más grande será su capacidad para escribir en ese idioma. La lectura y la escritura se complementan de manera tal que su enseñanza en forma conjunta le dan al instructor de idiomas la oportunidad de crear una atmósfera de clase más dinámica y a la estudiante la oportunidad de practicarlas en un ambiente caracterizado por la comunicación oral y escrita de sus sentimientos, opiniones y valores.
\end{abstract}

Palabras clave: La enseñanza de las lenguas extranjeras, lectura y escritura, interacción en clase, comunicación oral y escrita

\begin{abstract}
The interaction between reading and writing has not been given the attention that it deserves in foreign language teaching. By the same token, both skills have been traditionally taught in a separate form which runs counter to the learner's acquisition of one of the skills or both. This study proposes that the more language students the larger his or her capacity to write in the foreign language. Reading and writing complement each other in such a way that they give the language instructor the opportunity to create a more dynamic classroom atmosphere and the learner the opportunity to practice the skills in an environment characterized by the oral and written communication of their feelings, opinions and values.
\end{abstract}

Key words: Foreign language teaching, reading and writing, classroom interaction, oral and written communication.

\section{Introducción}

Tradicionalmente los profesores de idiomas han considerado a las destrezas de lectura y escritura como dos entidades separadas. Por lo tanto, por muchos años ambas destrezas han sido enseñadas de esa forma y se les ha considerado procesos que no se complementan el uno con el otro. Sin embargo, una cantidad apreciable de estudios de investigación señala que el aprendizaje de la escritura va paralelo al aprendizaje de la lectura y que una destreza puede ser usada para reforzar el aprendizaje de la otra. Antes de la aparición de las metodologías comunicativas para la enseñanza de los idiomas, se creía que la lectura era útil para la explicación de los patrones gramaticales. De hecho, un análisis de los textos para la enseñanza del inglés de los años sesenta y setenta muestra que los pasajes de lectura se incluían, por lo general, al final de la unidad y eran por demás muy cortos. Los ejercicios de comprensión de lectura consistían de escogencia múltiple, falso y verdadero o respuestas a preguntas. En otras palabras, la lectura era considerada como una destreza pasiva y su tratamiento en clase por lo tanto era poco significativo para la adquisición del segundo idioma. 
En el presente, y a raíz del auge comunicativo para la enseñanza de los idiomas, la lectura no solamente es considerada vital para proveer a los estudiantes de conocimiento general, sino que es un punto de partida para lograr un rendimiento óptimo a la hora de poner en práctica la destreza de escritura. La lectura complementaria sobre el tópico a desarrollar por escrito ayuda al estudiante a ser más profuso, pues la lectura utilizada para tal fin se convierte en una plataforma por medio de la cual puede expandir sus ideas, aumentar el vocabulario, utilizar estructuras gramaticales más avanzadas. En última instancia, la lectura es una fuente de inspiración para el proceso de escritura. Sin embargo, eso no ha sido siempre así. Para Grabe (2003) la relación entre la lectura y la escritura se ha enfocado en la escritura, pues es común que el aprendizaje logrado a través de los textos de lectura se vea reflejado en la escritura. Es menos común asumir que los estudiantes, y según Grabe, la mayoría de la gente, escriba para leer.

Cuando se propone que la integración de la lectura y la escritura ayuda a los estudiantes de idiomas a desarrollar destrezas lingüísticas, esto significa que ambas destrezas interactúan en un proceso por el que ellos adquieren el conocimiento necesario para leer y escribir en el segundo idioma con éxito. Según Brown (1994), la integración de estas destrezas requiere de un planeamiento cuidadoso, razón por la cual el instructor debe diseñar las actividades y técnicas de acuerdo al nivel de los estudiantes y sus necesidades de aprendizaje. Asimismo, el instructor debe tener una idea clara de los alcances de la lectura y escritura, cómo funcionan y cuáles son los procesos mentales involucrados cuando los estudiantes interactúan con el texto, leen o escriben. Los estudiantes deben ser motivados a experimentar con la lectura y escritura como medio para mejorar sus habilidades lingüísticas y como experiencia placentera para el aprendizaje del segundo idioma y el conocimiento general.
Cuando se trata de aprender un segundo idioma a través de la instrucción formal, la lectura y la escritura no juegan un papel menos predominante que la escucha y el habla. Con la introducción de la metodología de proceso en la enseñanza de la escritura, las destrezas de lectura y escritura no se han considerado más como independientes la una de la otra. Para Kroll (1991), el paradigma tradicional para las clases de escritura estaba basado en que los estudiantes leyeran un texto, lo discutieran y posteriormente escribieran sobre su contenido. Aunque es cierto que la escritura implica la acción de codificar ideas, y que por su parte la lectura implica la acción de descodificarlas, Kroll sugiere que la lectura es más bien un punto de partida para la escritura. Esta investigadora critica la filosofía dominante en métodos antiguos de enseñanza de los idiomas por medio de los cuales el estudiante aprende a escribir escribiendo y que por lo tanto la lectura tenía poco o nada que ver con la adquisición de la escritura.

Con respecto al paradigma tradicional que ha dominado la escritura por mucho tiempo, Kroll argumenta que la transformación en la enseñanza de la escritura ha sido el cambio de un enfoque en el producto de la escritura (paradigma tradicional) a un enfoque en la escritura como un proceso de desarrollo de ideas. A este respecto, Kroll invita a los profesores de inglés a ofrecer cursos que provean a los estudiantes con un repertorio de estrategias para la escritura de textos, ya sea a nivel del párrafo o a nivel del ensayo, que a la vez faciliten la comprensión de los objetivos y propósitos de la comunicación escrita. En su opinión, la lectura y la escritura deben dejar de ser destrezas pasivas que, por el contrario, en su papel de destrezas activas, promuevan la habilidad del estudiante de comunicarse, no sólo a nivel del texto discurso, sino que también a nivel del discurso hablado.

La lectura por su parte brinda propósitos prácticos a aquellos estudiantes que tienen un nivel menor de fluidez en el 
idioma. Los textos de lectura muestran, por ejemplo, modelos de lecturas en lengua inglesa los cuales proveen información lingüística que les ayuda a desarrollar conciencia sobre diferentes estilos de prosa y estrategias de escritura que posteriormente pueden poner en práctica a la hora de escribir. Por lo tanto, el trabajo escrito de estos estudiantes anticipa las necesidades de el lector y llena sus expectativas y aprenden que no están escribiendo para sí mismos, y que en última instancia, la interacción entre su texto y un lector tomará lugar en el futuro. En el caso particular de la clase de inglés como lengua extranjera, el instructor se convierte en lector o audiencia esperada del estudiante.

\section{Leer bien para escribir bien}

La lectura pone al estudiante de escritura en posición de predecir, en la medida de lo posible, lo que el lector desearía obtener del texto. Tal predicción ayuda al instructor a buscar materiales de lectura útiles para el buen desarrollo del texto escrito. En virtud de lo anterior, Hughey y otros (1983) señalan que el instructor debe desarrollar estrategias apropiadas de lectura en clase, de manera tal, que el proceso de lectura llegue a tener la efectividad necesaria para facilitar la escritura. Entonces, como destreza adquirida, la lectura se convierte en fuente de información lingüística permanente cada vez que el estudiante emprenda el proceso de escritura.

Como se puede ver, una de las más importantes contribuciones de la lectura en el desarrollo de la escritura es dar al estudiante ideas para escribir. Los estudiantes leen para reunir datos de otras fuentes de lectura y los aplican a sus propios escritos para analizarlos, pulirlos y asegurarse de que éstos cumplan con los requisitos que demanda la estrategia de lectura en la que se desarrolla el tema -narración, persuasión, y argumentación, entre otros-. A la vez, la capacidad de leer con comprensión promueve una dinámica interesante y de gran provecho en la clase de idiomas por medio de la cual los estudiantes leen, comentan y critican el trabajo escrito de sus compañeros con el fin de refinarlo. Esta actividad promueve la lectura del texto del compañero con eficacia y responsabilidad.

La lectura también ayuda a los estudiantes en aspectos que son útiles para la escritura efectiva. Por ejemplo, pueden mejorar su competencia en ortografía, aumentar su nivel de vocabulario, observar la organización de ideas y aprender a que el contenido de su escrito sea del entendimiento del lector. A medida que leen materiales en el segundo idioma, los estudiantes adquieren conciencia de cómo se presenta y ordena la información en un texto. Aprenden cómo están conectadas las ideas a través del vocabulario específico y evalúan las relaciones que unen el significado de las oraciones en el discurso escrito. Aprenden la relación entre la oración tópico y las oraciones de soporte, así como el papel que desempeña la conclusión, ya sea en forma de resumen, predicción, o solución del problema. No menos importante es la oportunidad que el texto de lectura da al estudiante para que comprenda las relaciones gramaticales entre los diferentes elementos de la oración: sujeto, verbo, predicado o complementos.

Algunos autores (Bernhardt, 1986; Carrell, Devine y Eskey, 1988; Earl, 1997) sugieren que la actividad de lectura se realice también en el hogar, pues fuera de clase la lectura tiene un efecto positivo en el desarrollo de esta destreza. Sin embargo, los instructores deben asignar lecturas que estén relacionadas con los temas que se discuten en clase. Al hacerlo, los instructores ponen en práctica su creatividad e influencia para que los estudiantes lean en forma extensa. Para lograr este objetivo, se necesita una selección bien definida de los tópicos de manera que el vocabulario permita a los estudiantes ampliar sus ideas y lograr una mayor comprensión del texto. La lectura en casa mantendrá el interés 
del estudiante en los tópicos de clase a la vez que aumenta su familiaridad con el vocabulario.

\section{La capacidad de leer}

La relación estrecha que existe entre la lectura y la escritura beneficia al estudiante de idiomas a medida que desarrolla habilidades en ambas destrezas. La integración de las dos destrezas debe formar parte de cualquier curso de escritura. $\mathrm{Su}$ desarrollo y práctica convierten al estudiante en participante activo en esta integración. Además, los instructores no deben dar énfasis solamente a los textos y tipos de actividades y técnicas usados en los cursos, sino que también deben observar en forma permanente los procesos que los estudiantes siguen cuando leen o escriben. A este respecto, la información que los estudiantes traen consigo a la hora de escribir es primordial y su aplicación al proceso de escribir debe tomarse muy en serio. Esta información, que llamaremos de fondo, permite a los estudiantes crear una conexión entre los textos de lectura y el desarrollo de las estrategias de escritura tanto en clase como en el hogar. Así, estarán en la capacidad de lograr un mayor grado de comprensión de los contenidos y tópicos y comunicarse en forma escrita más efectivamente.

La investigación en lectura y escritura ha dado como resultado importantes señalamientos sobre cómo ambas destrezas son adquiridas por los estudiantes. Por ejemplo, Carson (1992) propone que la habilidad para la lectura se transfiere más fácilmente de la lengua materna a la segunda lengua que la habilidad para la escritura. Según Carson, esto ocurre porque la lectura no está tan llena de convencionalismos como la escritura. Cuando una persona lee, usa una serie de técnicas que le permite "digerir" el material de lectura. Puede, por ejemplo, usar técnicas de pre-lectura, visualizar el texto o darle una hojeada antes de empezar con la lectura en sí. Por otro lado, no tiene que preocuparse de una audiencia y puede darle al texto su propia interpretación. Al contrario, cuando una persona escribe, debe tener en mente técnicas y estrategias de escritura muy claras y obtener retroalimentación durante todo el proceso.

\section{Evaluación y retroalimentación}

Leki (1991) sostiene que los estudiantes a menudo se quejan de los procedimientos de evaluación de los textos que producen. Le temen a las composiciones porque los instructores acostumbran calificar los trabajos como si estuvieran en un proceso de búsqueda de errores que se deben marcar con tinta roja. Al actuar así, los instructores dejan atrás su papel de facilitadores del proceso de escritura. Es decir, su preocupación ahora es el texto escrito como producto final del proceso y no cómo el estudiante llegó a producirlo. Es aquí donde la lectura cumple un papel muy importante en la construcción del texto escrito. La metodología de proceso le da la oportunidad al instructor de supervisar el trabajo del estudiante durante el proceso por el cual construye un texto. El instructor brinda retroalimentación y hace comentarios sobre los distintos componentes de la escritura. El instructor guía a sus estudiantes a ser lectores interactivos con el propósito de enriquecer el texto con ideas de lecturas afines al tópico. El papel del instructor deja de ser el de un corrector de trabajos para dar paso al de un "facilitador" en el proceso de escribir.

La retroalimentación debe darse durante el periodo de composición del texto escrito, no al final. Al respecto, cabe mencionar otro tipo efectivo de retroalimentación: la que proviene de los compañeros de clase. Compete al instructor proveer los lineamientos para que la participación de los estudiantes en el proceso de revisión del texto escrito producido por sus compañeros 
sea efectivo. Para ello, es preciso que la actividad esté basada primordialmente en (1) el enfoque del tema por parte del estudiante-escritor, (2) la organización de las ideas, (3) el uso efectivo de las estructuras gramaticales, y (4) la escogencia de vocabulario adecuado. Los comentarios de los estudiantes también debe enfocarse hacia la puntuación, el uso de mayúsculas, y las referencias bibliográficas. La experiencia dicta que en el curso de escritura la retroalimentación por parte de los compañeros de clase lleva varias sesiones de práctica durante el semestre para lograr mejores resultados.

\section{Estudiantes lectores y escritores}

Como lectores visualizamos al escritor como si nos hablara. Como escritores visualizamos al lector como si le habláramos. La lectura y la escritura intuyen la comunicación de pensamientos específicos con una audiencia específica. Para darse a entender en cualquiera de ambas destrezas, la persona valora el conocimiento previo de la audiencia. En ocasiones, la audiencia no tiene necesariamente que aceptar los argumentos que se le exponen. Por esta razón, el lector o autor debe tener en mente el interés de su audiencia ante el tópico en el cual se basan la composición o el ensayo. Como lectores, los estudiantes deben ser guiados a visualizar al autor e inferir sus motivos, tono y punto de vista como una manera de contribuir a que su texto tenga sentido. A este respecto, la lectura de un texto conlleva, por parte de los estudiantes, la identificación del propósito e intención del autor. De esta manera, la comunicación entre el autor y el estudiante-lector cobra sentido.

Los buenos hábitos de lectura se transfieren de la lengua nativa a la lengua extranjera. Por esta razón, entre más haya leído un estudiante en la primera lengua mayor será su oportunidad de convertirse en un buen lector en la segunda lengua. Es de esperarse entonces que el desarrollo efectivo de la lectura en su instrucción formal ayudará al estudiante a determinar con éxito el texto escrito, su significado y mensaje en su propia lengua o en la que aprende. En consecuencia, Eskey (1997) sostiene que enseñar la destreza de lectura a estudiantes que no traen el hábito de lectura bien desarrollado a través de la enseñanza formal será más difícil para los instructores de lenguas extranjeras. Por el contrario, conviene motivar al estudiante a leer en la clase de idiomas lo que necesite leer y en grandes cantidades.

Frank Smith (1988), experto estadounidense en el desarrollo de hábitos de lectura, propone que al convertirse en lectores de textos en una lengua extranjera los estudiantes se unen a la gente que lee en esa lengua como si se tratase de obtener la membresía a un club literario. Sin embargo, esta oportunidad requiere de práctica en la clase de idiomas para lograr la precisión en la lectura. Y para lograr este objetivo, Krashen (1993) propone que los instructores de idioma traten a sus estudiantes como colegas lectores interesados en la lectura y los motiven a ver cómo esta influye en sus vidas.

\section{Conocimiento de fondo}

Los estudiantes de idiomas tienen dos fuentes principales para construir un sistema conocido como segunda lengua. En la transferencia de información de un sistema a otro, la habilidad para leer se transfiere de forma más fácil de la primer lengua a la segunda lengua que la habilidad para escribir. Por lo tanto, según estos autores, habilidad de escribir puede y debe ser usada en la segunda lengua. Esto implica que existe una relación entre leer en la primera lengua y leer en la segunda lengua. Sin embargo, Carson (1992) advierte a los instructores que no deben depender de la transferencia automática de las destrezas de lectura de la lengua materna a la segunda lengua. Por esta razón, es necesario hacer énfasis 
en las destrezas de lectura en la clase de idiomas, sobre todo en niveles de enseñanza altos, donde la información lingüística puede ser el recurso más significativo para desarrollar las destrezas del lenguaje.

En relación con la enseñanza de las destrezas de lectura, Aebersold \& Field (1997) discuten tres estrategias esenciales que los estudiantes necesitan tener en cuenta antes de interactuar con un texto escrito. La primera estrategia consiste en establecer un propósito para que se dé la lectura. Esto, según ambos autores, ayuda a que los estudiantes ajusten sus estrategias de lectura y expectativas de acuerdo al texto que van a leer. La segunda estrategia consiste en activar y construir conocimiento de fondo, lo cual es de suma importancia porque el conocimiento sobre el tópico hará más efectiva la comprensión del texto. A través del conocimiento de fondo, los estudiantes pueden predecir, anticipar y relacionar la información en el texto. En la tercera estrategia, el estudiante visualiza el texto para establecer expectativas reales acerca de éste. De esta manera, los estudiantes pueden leer con un nivel más alto de comprensión.

El desarrollo de destrezas de lectura como las propuestas incidirá en una mejor aplicación del conocimiento adquirido a la hora de desarrollar las ideas en el texto escrito. Es decir, la adquisición de destrezas de lectura puede conducir al éxito en el proceso de escritura. Para tal fin, es necesario que los estudiantes sean guiados hacia la comprensión efectiva del texto, sobre todo la construcción del significado correcto de las ideas en la lectura. La labor del instructor es clave para evaluar la comprensión por medio de preguntas, elaboración, confirmación y rechazo de hipótesis sobre el contenido de la lectura. Si las estrategias fallan, el instructor debe guiar a los estudiantes a reparar la información incorrecta que se haya derivado del error para así continuar de nuevo con el proceso de lectura.
Es importante hacer énfasis en el uso del conocimiento de fondo como preludio para una efectiva comprensión de lectura. Este conocimiento promueve la interacción entre lector y texto con lo cual el estudiante trae al proceso de lectura aquellas experiencias de su vida que sean afines al contenido del texto. Estas experiencias son diferentes de acuerdo a cada estudiante quien las incorpora a su aprendizaje en sus relaciones con la familia, la comunidad, la escuela, la sociedad y la cultura. Además, los estudiantes leen un texto haciendo uso de su experiencia con diferentes tipos de textos: periódicos, revistas, folletos, lecturas usadas en distintas clases que a su vez pertenecen a diferentes áreas del conocimiento para las que diferentes tipos de interacción fueron necesarios. Por esta razón, la interacción entre lector y texto cambia constantemente. Sin embargo, tras ella, el estudiante posee ahora el conocimiento de fondo clave para abocarse al proceso de escritura.

\section{Los textos de lectura}

Cuando una persona escribe, debe tener en mente a una audiencia y respetar ciertas convenciones que le dan el marco a su trabajo. El texto que esta persona produce estará expuesto a diferentes interpretaciones, dependiendo de la experiencia de fondo y las habilidades lingüísticas de la persona. En la clase, los instructores deben concienciar al estudiante de que la lectura es un acto interno, pero que al escribir lo hacen para una audiencia. Se puede inferir entonces que la habilidad de escribir es más difícil que la habilidad de leer. Sin embargo, la lectura se convierte en una herramienta importante en el desarrollo de la escritura.

La lectura en la clase de idiomas constituye una ayuda para el desarrollo de la escritura, pues da al estudiante la información lingüística necesaria para crear sus propios textos. En consecuencia, si la 
información lingüística que los estudiantes reciben es efectiva, el resultado será una buena pieza de escritura. Esta relación entre lectura y escritura es también apoyada por Krashen (1984) quien asegura que la competencia en la escritura de los estudiantes de lenguas extranjeras se deriva de la lectura de grandes cantidades de texto que ellos están motivados para leer o que leen por placer. De ahí que una de las labores del instructor es escoger lecturas que promuevan esa motivación e interés en aras de una ulterior aplicación del conocimiento obtenido a través de la lectura cuando llegue el momento de elaborar el texto escrito.

Si la lectura tiene una influencia positiva en la escritura, es necesario entonces contar con una serie de actividades por medio de las cuales los estudiantes, como lectores, tengan la oportunidad de responder a preguntas sobre el contenido de las lecturas. Por ejemplo, clarificar los contenidos de un pasaje para lo cual el instructor puede hacer uso de un examen corto acompañado de una discusión del contenido de las preguntas. En el caso de un cuento, por ejemplo, el instructor puede evaluar la comprensión a través de un ejercicio de escogencia múltiple y brindar retroalimentación al revisarlo. También, el instructor puede distribuir dos textos cortos con afinidad en el tópico y hacer preguntas sobre los puntos de coincidencia o contraste de los autores. Asimismo, es posible leer una parte o un capítulo corto de un libro cuyo nivel de lectura esté al alcance de la comprensión de los estudiantes, y luego discutir su contenido con base en las ideas principales, punto de vista y detalles que apoyan la posición del autor.

Al poner en práctica el conocimiento adquirido a través de la lectura, el estudiante puede desarrollar la capacidad de ahondar aún más en el tópico asignado y encauzar sus ideas hacia el texto escrito. Se puede hablar entonces de una activación de su esquema mental por medio de lo cual la lectura funcionará como plataforma de despegue hacia el proceso de escritura. Sin embargo, el proceso no se lleva a cabo en forma espontánea. Por ejemplo, Hughey y sus colegas (1983:62) sugieren técnicas de lectura del tipo "heurístico", las cuales activan la memoria y provocan la imaginación. Entre estas técnicas se encuentran: (1) el desarrollo de un tópico de acuerdo a tópicos relacionados, por ejemplo, los alimentos y su relación con la salud, (2) la limitación de un tópico, por ejemplo, el tópico de la energía se puede limitar a tres áreas: solar, nuclear y eólica, (3) la generación de ideas, por ejemplo, la discusión de los alcances de un tratado entre dos naciones, y (4) la relación y ordenamiento de ideas, por ejemplo, los pasos incluidos en el proceso de reconstrucción de una región después de un terremoto.

Es posible que el estudiante no esté acostumbrado a trabajar con un texto por medio del uso de estas actividades en cursos en su lengua natal. De acuerdo con Winer (1992), esta es una de las razones por las cuales los estudiantes tienen experiencias frustrantes al escribir en la lengua término. Es obvio que esta limitación puede interferir también con la escritura en la clase de idiomas. En consecuencia, antes de empezar a escribir en la segunda lengua, los estudiantes deben ser expuestos a actividades de lectura que en sí son actividades de pre-escritura. En el evento de que su experiencia con la escritura en su propia lengua no haya sido la mejor, la guía y retroalimentación por parte del instructor de idiomas en el proceso de leer hará que su frustración sea menor a la hora de enfrentar el proceso de escribir en el segundo idioma. Lógicamente, para que el proceso sea completo, será necesario poner en práctica actividades de pos-escritura para revisar y reforzar el contenido de lo que se ha escrito. Esto intuye también una interacción posterior entre el producto escrito, sus autores -los estudiantes-y el instructor quien provee retroalimentación. 


\section{La influencia de la primera y segunda lenguas}

Estudios realizados por Lopes han identificado también procesos cognitivos comunes y/o componentes estructurales detrás de la lectura y la escritura. Estos procesos se asemejan a los propuestos por los transformacionalistas en la década de los años setenta y que fueron denominados "estructura profunda del lenguaje". En virtud de estos procesos, Eisterhold y otros (1990) proponen que la práctica con una destreza puede conducir a progresos significativos en otra, si existe una interacción metodológicamente bien elaborada. Por otro lado, en un estudio con niños cuya lengua nativa es el español Freeman \& Freeman (1997) han demostrado que estos niños pusieron en práctica sus conocimientos del español para la adquisición de una serie de destrezas de lectura y escritura en inglés. A través del uso de ejemplos tomados en sus clases, ambos investigadores critican la enseñanza tradicional de la lectura y escritura españolas. Con base en su estudio, recomiendan métodos para el desarrollo de las dos destrezas con piezas de literatura infantil. Muchos de los ejemplos en su estudio muestran la capacidad natural de los niños para el desarrollo de la lectura y escritura.

Cuando el estudiante lee en el segundo idioma, extrae información del texto de acuerdo al propósito asignado a ese texto y a su motivación e interés por leer. A su vez, el estudiante provee en forma subconsciente información para que la lectura tenga sentido. Este tipo de información se denomina "información de fondo", o conocimiento global, adquirido a través de la experiencia en el medio lingüístico natural, es decir, el primer idioma, o en el entorno educativo. Durante el proceso de lectura en el segundo idioma, ambas fuentes de conocimiento lingüístico, meta y término, se combinan para dar sentido al contexto.

Estudios realizados en la interacción lectura-escritura en la adquisición tanto de la primera como la segunda lengua realizados por Carson y Leki, 1993) indican que el conocimiento de fondo derivado de la lectura se transfiere con mayor facilidad en el caso de la escritura en la segunda lengua. Es por esta razón que comúnmente se sostiene que los buenos lectores en la primera lengua tienen grandes oportunidades de ser buenos escritores en la segunda. Estos estudios señalan además a la lectura como punto de partida para la escritura y no a la inversa (Krashen, 1984). Incluso, Thompson (2001) señala que, para el desarrollo de las destrezas de escritura, no basta la sola lectura de textos relacionados con el tópico. Este investigador sugiere el análisis del discurso, técnica con la cual reporta un beneficio en particular al guiar a sus estudiantes a explorar cómo funciona un texto en particular.

Al preceder el proceso de escritura la lectura provee retroalimentación al estudiante-escritor, lo que redunda en un mejor desempeño a la hora de construir su escrito, en especial a nivel del párrafo y del ensayo. $\mathrm{Al}$ integrar apropiadamente las destrezas de lectura y escritura, los instructores de idiomas logran tres objetivos importantes: (1) conducen a sus estudiantes a la adquisición de éstas, (2) estimulan a los estudiantes a leer, (3) hacen conciencia entre ellos de que a mejores lectores mejores escritores, y en última instancia, (4) contribuyen a enriquecer su conocimiento de fondo con información útil para el futuro.

\section{Conclusión}

La enseñanza de la lectura conlleva un proceso de adaptación por parte del instructor según sea el nivel de dominio del segundo idioma de sus estudiantes. Este proceso es crucial en la adquisición de las estrategias de comprensión del texto, pues se espera que los estudiantes lo entiendan sin uso continuo del diccionario. Por ejemplo, si el objetivo de una actividad es resumir una lectura, éste no se podrá lograr si 
los estudiantes no poseen un grado razonable de comprensión de los contenidos del texto de lectura. De ahí la importancia de las actividades de pre-lectura, las cuales no solamente estimulan al estudiante a poner en práctica su conocimiento de fondo, sino que le permiten manejar información sobre el tema específico a desarrollar en forma escrita. De esta forma, el contenido de las lecturas tiene sentido y cobra interés por parte de los estudiantes a la hora de escribir.

Además de brindarle a los estudiantes información útil acerca del tópico sobre el cual van a escribir, las lecturas complementarias contienen aspectos de forma: estilos, estrategias de escritura y tipos de discurso. En consecuencia, la selección de una o varias lecturas relacionadas con un tópico en particular permite al estudiante un modelo de la estrategia de lectura a emplear en su composición o ensayo. La utilización de estas lecturas antes de la actividad de escritura provee también al estudiante términos de vocabulario y expresiones idiomáticas que pueden enriquecer el texto escrito.

Se puede concluir que la escritura en cierta forma la lectura confirma el nivel de comprensión de textos. Por tal razón, en cursos modernos de composición y retórica la lectura es la forma más frecuente de retroalimentación para incorporar ideas al texto escrito. Esto se logra a través del trabajo con una lectura o lecturas como complementarias como modelos, las cuales discuten el mismo tópico sobre el cual los estudiantes van a basar sus escritos. Dado que en este caso el énfasis en la lectura conlleva un objetivo primordial: enriquecer el proceso de escritura, Lopes (1991) considera que las estrategias efectivas de lectura tendrán efectos positivos en la adquisición de la escritura en el segundo idioma. El resultado será más productividad y creatividad al escribir.

La enseñanza moderna de la lectura debe considerar el tiempo como elemento básico para su desarrollo por parte del estudiante. Para escribir con un buen grado de precisión es necesario que el estudiante dedique tiempo a lecturas relacionados con el tópico de escritura. En el proceso, la labor del instructor es crucial en cuanto a dar retroalimentación sobre la incorporación de los materiales leídos en el contexto apropiado. Esto incluye el uso correcto de mecanismos de transición de manera tal que la incorporación del material leído en el texto sea parte de la secuencia lógica de ideas y no piezas de texto sin sentido en el párrafo. Como se puede notar, el factor tiempo es importante para lograr una relación idónea entre ambas destrezas. Por otro lado, la escritura es pues un proceso complejo, no una destreza aislada, como se le consideró en décadas pasadas. Este proceso incluye actividades de pre-escritura, revisión del texto y re-escritura, y en su desarrollo la incorporación de ideas y temas provenientes de lecturas relacionadas es fundamental para que la productividad creatividad por parte de los estudiantes sean reflejadas por parte del estudiante en el texto escrito.

\section{Referencias bibliográficas}

Aebersold, Jo \& Mary Lee Field. From reader to writing teacher. Cambridge: Cambridge University Press. 1997.

Bernhard, E. B. Reading in the Foreign Language. Listening, reading and writing: Analysis and application. D. H. Wing (editor).Middlebury, Vermont: Northeast Conference. 1986.

Brown, H. Douglas. Teaching by principles: An interactive approach to language pedagogy. New Jersey: Prentice Hall Regents. 1994.

Carrell, Patricia L., J. Devine \& D. Eskey (editores). Interactive Approaches to Second Language Reading. Cambridge: Cambridge University Press. 1988. 
Carson, Joan \& Ilona Leki (editoras). Reading in the composition classroom. Second language perspectives. Boston : Heinle \& Heinle. 1993.

Carson, Joan. Becoming biliterate: First Language Influences. Journal of Second Language Writing. (1), 37-60. 1992.

Earl, Donna. Learning to Love Reading: Focus on Basics. 1(B). En línea. 1997.

Eisterhold Carson, Joan. P. Carrell, S. Silberstein, B. Kroll \& P. Kuehn. Reading- Writing Relationships in First and Second Language. TESOL Quarterly. 24, 245-266. 1990.

Eskey, David. Models of Reading and the ESOL Student: Implications and Limitations. Focus on Basics. (1), 3. Disponible en línea: http:gseweb. harvard.edu/-ncall/fob/1997/ eskey.htm. 1997.

Freeman, Yvonne S. \& David E. Freeman. Teaching Reading and Writing in Spanish in the Bilingual Classroom. Porstmouth, New Hampshire: Heinemann. 1997.

Grabe, William. Reading and writing relations: Second language perspectives on research and practice. Exploring the Dynamics of Second Language Writing. B. Kroll (editora). Cambridge: Cambridge University Press. 2003.

Hughey, Jane B., Deanne R. Wormuth, V. Faye Hartfield \& Holly L. Jacobs Teaching ESL Composition: Principles and Techniques. Rowley, Mass.: Newbury House Publishers, Inc. 1983.

Krashen, Stephen. Writing: Research, Theory and Applications. Oxford:
Pergamon Institute of English. 1984.

Krashen, Stephen. The Power of Reading. Englewood, Colorado: Libraries Unlimited. 1993.

Kroll, Barbara. Second Language Writing. Cambridge: Cambridge University Press. 1991.

Leki, Ilona. Twenty-five years of contrastive rhetoric: Text analysis and writing pedagogies. TESOL Quarterly. 25, 123-143. 1991.

Lopes, Dalila. From Reading to Writing Strategies. English Teaching Forum. (4), 29, 42-44. 1991.

Nation, Paul. The Language Learning Benefits of Extensive Reading. Language Teacher. (21), 5, pp. 13-16. 1997.

Nelson, Nancy \& Robert C. Calfee (editores). The Reading and Writing Connection: Ninety - Seventh Yearbook of the National Society for the Study of Education, Part II. Chicago: University of Chicago Press. 1998.

Smith, Frank. Joining the Literacy Club. Porstmouth, New Hampshire: Heinemann. 1988.

Thompson, Geoff. Interaction in Academic Writing: Learning to Argue with the Reader. Applied Linguistics. (22) 1, pp. 58-75. 2001.

Winer, Lise. Spinach to Chocolate: Changing Awareness and Attitudes in ESL Writing Teachers. TESOL Quarterly. (1) 26, pp. 57-80. 1992. 